\title{
МИРОВОЙ ОПЫТ СТРАН КРЕДИТОВАНИЯ МАЛОГО И СРЕДНЕГО БИЗНЕСА
}

\section{WORLD EXPERIENCE OF COUNTRIES LENDING TO SMALL AND MEDIUM-SIZED BUSINESSES}

\section{Shaydullina}

Summary. The article is devoted to the consideration of foreign experience related to the development and support of medium and small businesses. The article analyzes the economic processes of the EAEU member states and summarizes the foreign practice of individual states. In conclusion, several of the most pressing problems in the field of lending to medium and small businesses in the EAEU member states are highlighted. The article has been prepared based on the results of research carried out at the expense of budgetary funds on the state order of the Financial University.

Keywords: subjects of medium and small business, medium and small business, indicators, EAEU member states, availability of credit resources, lending.

\author{
Шайдуллина Венера Камилевна \\ К.ю.н., дочент, Финансовый университет при \\ Правительстве Российской Федерации (г. Москва) \\ VKShajdullina@fa.ru
}

Аннотация. Статья посвящена рассмотрению иностранного опыта, связанного с развитием и поддержкой субъектов среднего и малого предпринимательства. В статье проанализированы экономические процессы стран-членов ЕАЭС и обобщена иностранная практика отдельно взятых государств. В заключении выделено несколько наиболее актуальных проблем в области кредитования субъектов среднего и малого бизнеса в странах-членах ЕАЭС. Статья подготовлена по результатам исследований, выполненных за счет бюджетных средств по государственному заданию Финансового университета.

Ключевые слова: субъекты среднего и малого предпринимательства, средний и малый бизнес, индикаторы, страны-члены ЕАЭС, доступность кредитных ресурсов, кредитование. ектор среднего и малого бизнеса выступает в качестве неотъемлемого элемента и стабилизатора рыночной экономики. Именно субъекты среднего и малого бизнеса обеспечивают материальное благосостояние населения и жизнеспособность экономики в условиях финансово-экономического кризиса. В западных странах средние и малые предприятия способствуют формированию конкурентной среды и повышают рыночную конъюнктуру. Странам с развивающейся экономикой будет полезен опыт развитых государств в данной сфере.

Многие предприятия сегмента среднего и малого бизнеса сталкиваются с проблемами, связанными с финансированием хозяйственной деятельности. Отечественный и иностранный опыт показывает, что одним из важнейших источников внешнего финансирования субъектов среднего и малого предпринимательства выступает банковское кредитование. Соответственно, вопрос доступности кредитования и кредитных ресурсов для средних и малых предприятий обладает сегодня повышенной актуальностью.

Аналитики и эксперты в области финансов все чаще говорят о предстоящем мировом финансово-экономическом кризисе. Их слова подтверждаются целым рядом факторов, в том числе постепенным снижением числа запускаемых инвестиционных проектов, падением спроса населения на товары длительного пользования, замедлением темпов роста экономики (национальной и мировой). Очень важно предпринять необходимые шаги по подготовке к предстоящим изменениям, разработав новый пакет антикризисных мер. В этот пакет обязательно необходимо включить меры по поддержке субъектов среднего и малого бизнеса.

К настоящему времени проведено немало теоретических исследований, посвященных анализу ситуации с кредитованием среднего и малого бизнеса в странах-членах ЕАЭС. В этих исследованиях затрагивается не только кредитование, но и иные актуальные вопросы (фискальная политика в странах ЕАЭС; принятие мер по улучшению бизнес-климата; нейтрализация административных барьеров; финансирование субъектов среднего и малого бизнеса из разных источников; государственная поддержка). Проанализировав отдельные публикации отечественных авторов и иностранные первоисточники, можно в общих чертах понять специфику кредитования средних и малых предприятий. Часто в литературе описываются и основные этапы кредитования. 
Обобщив иностранную практику стран-членов ЕАЭС (России, Республики Беларусь, Республики Армения, Кыргызстана и Республики Казахстан) в сфере кредитования среднего и малого бизнеса в кризисных условиях, можем сделать некоторые выводы. Так, в 2015-2016 гг. средние и малые предприятия, функционирующие в странах ЕАЭС, сталкивались с разными, но похожими по характеру проблемами (неадекватно завышенная стоимость кредитных ресурсов; спад внутреннего спроса; отсутствие государственной поддержки на достаточном уровне и др.). Такая ситуация была обусловлена депрессивностью национальных экономик стран-членов ЕАЭС, а также целым рядом субъективных и объективных причин. Но уже в 2017 г. на макроэкономическом уровне произошли определенные изменения, следствием чего стало улучшение бизнес-среды и внешнеторговой конъюнктуры. Также с 2017 года началось снижение процентных ставок по кредитам в национальной валюте. В 2018 году тенденция снижения процентных ставок распространилась и на валютные кредиты.

Проведенный анализ показал наличие относительно развитой системы государственной поддержки во всех странах-членах ЕАЭС. Существующей системой предусмотрено льготное финансирование и кредитование государственного сектора. Несмотря на это, в период с 2014 по 2016 гг. наблюдалось значительное сокращение численности занятых на предприятиях государственного сектора. Такая ситуация в разной степени имела место во всех странах ЕАЭС. По словам экспертов, рабочая сила, массово высвобождаемая из государственного сектора в связи с кризисом в экономике, могла бы найти применение в частном сегменте. Но для этого нужны более благоприятные условия в части обеспечения доступности кредитных ресурсов. Без создания надлежащих условий для финансирования и развития сегмента среднего и малого бизнеса рассчитывать на эффективное задействование рабочей силы явно не приходится.

В контексте настоящего исследования целесообразно рассмотреть базовые индикаторы развития и обеспечения доступности кредитных ресурсов для средних и малых предприятий. При анализе базовых индикаторов необходимо учитывать, что условия, влияющие на роль и место субъектов среднего и малого бизнеса в национальной экономике, варьируются не только в зависимости от государства. Эти условия могут различаться и по финансовым организациям одной юрисдикции. Приблизительно треть иностранных государств четко придерживается рекомендаций ОЭСР, сформулированных в рамках конференции 2004 г., прошедшей в Турции. В соответствии с этими рекомендациями, к числу средних, малых и микропредприятий относятся бизнес-субъекты, в которых менее 250 наемных работников.
Обязательное условие - предприятие должно быть зарегистрировано в соответствии с действующим законодательством. Указанный критерий признается во всех европейских странах. В Соединенных Штатах для того, чтобы быть причисленной к сегменту среднего и малого бизнеса, компания должна проходить по двум условиям:

1. Во-первых, размер выручки в год должен быть не более 250 миллионов долларов.

2. Во-вторых, максимальное количество работников в компании должно составлять 500 чел. [1].

В некоторых странах достаточно соответствия всего одному условию (численность персонала, объем выручки или сумма инвестиций в предприятие). Так, в Индии к указанному сегменту относятся предприятия, размер инвестиций в которые не превышает 0,8 млн. долларов (сфера услуг) или 1,61 млн. долларов (производство). А в Китае крупным считается предприятие, на котором работает от 1501 чел. Соответственно, если численность работников меньше, то компания входит в число субъектов среднего и малого бизнеса [3].

Международная организация труда, активно занимающаяся развитием социального диалога и занятости, а также продвижением национальных программ достойного труда, в одном из своих официальных докладов обратила внимание на то, что на долю среднего и малого бизнеса приходится более 2/3 занятых. Эти цифры свидетельствуют о том, что именно данный сектор обеспечивает значительную часть производства мирового ВВП в странах с развивающейся и развитой экономикой. Аналогичные выводы представлены в документах Европейского Центрального Банка (The European Central Bank - ECB), Европейской комиссии (European Commission - EC) и Всемирного банка (The World Bank) [4].

Исследование глобального масштаба, проведенное МОТ в 2005 году, показало, что доля субъектов среднего и малого бизнеса составляет 90\%. Соответственно, на крупные предприятия приходится только 10\%. Это показатели по всему миру. По данным этого же исследования, в развитых и развивающихся странах на средние и малые предприятия приходилось 63\% и 34\% трудоустроенных соответственно. Причем, если учитывать теневой сектор, то реальные цифры могут быть и больше.

Средние и малые предприятия в 2013 году обеспечивали рабочими местами более $2 / 3$ трудоспособной части населения в странах ЕС. При этом из 100\% официально зарегистрированных предприятий около 99\% относились к рассматриваемому сегменту [1].

По мнению некоторых аналитиков, число субъектов среднего и малого бизнеса в стране зависит от уровня 
развития национальной экономики, а остальные факторы играют второстепенную роль [2]. В исследовании R. Ramalho, M.L. Mirmulstein и K.Kushnir, проведенном в 2010 г., рассмотрены данные по плотности средних и малых предприятий во всем мире за 2007 год. Россия вошла в число стран, в которых на тысячу чел. населения приходится от одного до десяти субъектов среднего и малого бизнеса. В эту же группу вошли такие страны, как Республика Беларусь, Грузия, Индия, КНР, Украина, Филиппины.

Положительная динамика развития субъектов среднего и малого бизнеса в странах с развивающейся и развитой экономикой обеспечивается благодаря доступности финансирования. Многие предприятия рассматриваемого сектора сталкиваются с трудностями в части эмиссии облигаций и вывода акционерного капитала напрямую на рынок. Во многом такие трудности обусловлены небольшими объемами производства и реализации продукции. Поэтому очень часто для средних и малых предприятий единственными источниками финансирования являются банковские кредиты и венчурное финансирование. Если применяются инструменты венчурного финансирования, то инвесторами оцениваются перспективы экономического роста бизнес-субъекта, уровень его предполагаемых доходов, а также доходность активов. В таком случае при анализе предприятия в дополнение к методам оценки, применяемым при долговом или акционерном финансировании, используются и иные методы.

В последние годы система кредитования субъектов среднего и малого бизнеса выступает одной из самых острых и актуальных тем. Ее исследованием занимаются не только международные организации (МОТ, ОЭСР, European Commission, The European Central Bank, МВФ, The World Bank), но и некоторые правительства. Результаты исследований затем закладываются в основу разработки мер поддержки субъектов среднего и малого бизнеса.

Начиная с 2008 г., European Commission и The European Central Bank на регулярной основе проводят исследования по вопросам определения степени доступности кредитов для субъектов среднего и малого бизнеса. Исследования проводятся более чем в 35 государствах, в т.ч. в 28 странах ЕС.

ОЭСР на ежегодной основе проводит анализ доступности кредитных ресурсов для средних и малых предприятий. В основу анализа заложено 13 ключевых показателей. К ним относятся:

1. Общее число банкротств с цифрами, отражающими динамику по бизнес-субъектам.

2. Общий объем коммерческого кредитования субъектов среднего и малого предпринимательства.
3. Объем краткосрочных кредитов (со сроком менее 12 месяцев) в общем объеме кредитов, выданных субъектам среднего и малого бизнеса.

4. Общий объем залоговых кредитов.

5. Объемы венчурного финансирования и объемы портфельных инвестиций.

6. Отсрочка по платежам с цифрами, отражающими динамику отсрочки в днях.

7. Общий объем кредитов, выданных с участием государственных гарантий.

8. Объем государственных гарантий, в т.ч. в денежном эквиваленте по кредитам, выданным средним и малым предприятиям.

9. Объем прямого кредитования из бюджета государства.

10. Показатель, отражающий разницу между ставками, установленными по кредитам для субъектов среднего и малого бизнеса, а также по кредитам для крупных предприятий.

11. Размер средневзвешенной ставки по кредитам для бизнес-субъектов рассматриваемого сегмента.

12. Соотношение между количеством поданных кредитных заявок и количеством удовлетворенных.

13. Соотношение объема просроченной задолженности по кредитам к общему объему выданных кредитов.

Вышеуказанные показатели и индикаторы отражаются в табличном виде и публикуются в виде статистики в разрезе стран, что позволяет отследить изменения в динамике.

С помощью этих индикаторов можно оценить фактор выживаемости рассматриваемого сегмента в кризисные периоды и периоды восстановления экономики; увидеть изменения в распределении объема кредитования по государствам, а также реальную структуру кредитов в части оборотного капитала, финансирования, инвестиций в основные фонды; сформировать представление о перспективах развития предприятия на долгосрочный период и о действующих условиях кредитования; оценить дополнительные источники финансирования для бизнес-стартапов; проследить финансовые потоки и проблемы, осложняющие процесс финансирования; определить уровень государственной поддержки средних и малых предприятий; оценить объемы государственной поддержки для финансирования рассматриваемого сегмента, а также увидеть реальные объемы прямого государственного финансирования для средних и малых предприятий; увидеть, как коррелируют ставки в зависимости от размера предприятия; увидеть взаимную связь между условиями по кредитам и премиальной платой за риск, взимаемой банками с предприятий рассматриваемого сегмента; 
Таблица 1. Объемы кредитования субъектов среднего и малого бизнеса в отдельных странах в 2010 г.*

\begin{tabular}{|l|l|l|}
\hline$№$ & Государство & Объем кредитования \\
\hline 1. & Эстония & 7 \\
\hline 2. & Казахстан & 9 \\
\hline 3. & Польша & 41 \\
\hline 4. & Индия & 53 \\
\hline 5. & Турция & 59 \\
\hline 6. & Бразилия & 61 \\
\hline 7. & Таиланд & 80 \\
\hline 8. & Российская Федерация & 84 \\
\hline 9. & Тайвань & 97 \\
\hline 10. & Бельгия & 107 \\
\hline 11. & Австралия & 156 \\
\hline 12. & Франция & 303 \\
\hline 13. & Нидерланды & 463 \\
\hline 14. & США & 700 \\
\hline 15. & КНР & 2110 \\
\hline
\end{tabular}

* Составлено автором

оценить готовностью финансовых учреждений кредитовать предприятия рассматриваемого сектора и увидеть связь между условиями по кредитам; оценить уровень финансовой дисциплины и определить степень кредитоспособности бизнес-субъектов [11].

Исследования, в основу которых заложены вышеперечисленные индикаторы, позволяют сформировать общее представление о политике государства в отношении сегмента среднего и малого бизнеса; оценить масштаб финансового кризиса; исследовать существующие в стране меры поддержки, предусмотренные для таких предприятий, а также увидеть, насколько они результативны. В настоящее время подобные исследования проводятся по 18 государствам. Особенность и ценность таких исследований в том, что они проводятся до кризиса, непосредственно в период кризиса и после его завершения, когда экономика вступает в фазу восстановления.

Оценка системы кредитования бизнес-субъектов рассматриваемого сегмента показывает наличие серьезных диспропорций в объеме между государствами с развитой и развивающейся экономикой. В соответствии с данными, опубликованными Всемирным Банком, в 2008-2011 гг. ежегодный объем кредитования рассматриваемого сегмента находится в пределах 10 трлн. долларов, что является довольно внушительной цифрой.

Если смотреть на показатель соотношения объема кредитования к ВВП, то он варьируется в диапазоне от 3\% (развивающиеся государства) до 13\% (страны с развитой экономикой).
В табл. 1 представлены объемы кредитования (в млрд. долларов США) предприятий рассматриваемого сегмента за 2010 год.

В рамках настоящей статьи необходимо рассмотреть пакет дополнительных мер по государственной поддержке кредитования и повышению доступности кредитов для субъектов среднего и малого бизнеса в странах ОЭСР в условиях рецессии. С этой целью взяты результаты расширенного сравнительного анализа. Это позволило определить уровень развития кредитования бизнес-субъектов интересующего сегмента, а также выявить страны, в которых наилучшие показатели в данной сфере. К числу таких стран относятся: Венгрия, Швеция, Италия, Словакия, Южная Корея и Португалия. Также была рассмотрена практика иностранных государств в части повышения доступности коммерческих кредитов для субъектов среднего и малого предпринимательства и доказано, что этот момент способствует снижению уровня безработицы в стране и положительно влияет на развитие национальной экономики.

В условиях финансово-экономического кризиса кредитная поддержка субъектов среднего и малого предпринимательства предоставляется по нескольким направлениям. Так, например, вследствие ужесточения условий кредитования субъектов среднего и малого бизнеса количество одобренных заявок на кредит в проанализированных странах значительно уменьшилось. Исследование, проведенное ЕК совместно с The European Central Bank, показывает, что в 2009 г. уровень неодобренных заявок по кредитам в странах 
ЕС достиг отметки в 18\% (ранее было зафиксировано значение в 12\%). Буквально через год этот показатель упал до $11 \%$. Правительства некоторых стран начали расширять программы поддержки и использовать дополнительные инструменты (прямое финансирование, расширение гарантийных обязательств и др.), что позволило компенсировать усложнение доступа к кредитам. К слову, в некоторых странах удалось добиться практически стопроцентного уровня обеспечения государственных гарантий по кредитам.

Помимо прочего, пакеты мер поддержки рассматриваемого сегмента включали: предоставление государственных гарантий при выдаче новых кредитов; бесплатное консультирование предприятий перед подачей заявки на кредит; введение новых кредитных продуктов (краткосрочные и контр циклические кредиты); более продолжительная отсрочка по платежам, если кредиты выданы в рамках реализации программ венчурного финансирования; снижение стоимости гарантийных схем [5].

В Дании гарантийные схемы наиболее активно использовались именно при кредитовании сегмента среднего и малого бизнеса, а также предприятий-экспортеров. Кредитные ресурсы необходимы предприятиям для пополнения оборотного капитала и в качестве инвестиционных инструментов. Экспортно-кредитное агентство Дании (Eksport Kredit Fonden) в 2010 г. могло предоставить гарантий на общую сумму 355,1 млн. долларов, что равнялось примерно 6\% от общей массы кредитов, выданных субъектам среднего и малого предпринимательства. В 2007 г. объем предоставленных государственных гарантий составлял 25,8 млн. долларов США, а в 2010 г.- 91,5 млн. долларов США.

В Италии разработкой мер по повышению доступности кредитных ресурсов для средних и малых предприятий занимается Центральный гарантийный фонд. В 2010 г. Фонд получил от государства более 5,2 млрд. евро, из которых на кредитование рассматриваемого сегмента было направлено почти 3,6 млрд. евро. В Венгрии в этом же году государственные гарантии покрывали примерно 12,6\% выданных ссуд.

Южнокорейское правительство увеличило поддержку рассматриваемого сегмента при первых же проявлениях глобального кризиса. В соответствии с программой государственного гарантирования, в 2007 г. размер государственных гарантий достиг отметки в 42,1 млрд. долларов. Через три года данный показатель составил 49,6 млрд. долларов.

В Швеции реализацией программы государственных гарантий занимались специализированные га- рантийные фонды, однако с 2010 г. программа была свернута. Гарантии предприятиям-экспортерам предоставляет кредитное экспортное агентство Швеции, а право обеспечивать сегмент среднего и малого бизнеса государственными гарантиями перешло к Шведскому банку развития (Swedbank).

В Словакии в 2007-2010 годах субъекты предпринимательства один раз в год получали доступ к кредитам в общей сумме около 70-80 млн. евро.

В Португалии субъектам среднего и малого бизнеса предоставлялась достаточно серьезная государственная поддержка в течение всего кризисного периода. В 2007 г. по государственным гарантиям было выдано кредитов на общую сумму 0,7 млрд. евро. Через три года данный показатель достиг отметки в 6,8 млрд. евро, т.е. налицо резкий рост. Экспортно-кредитное страхование, благодаря которому удалось обеспечить отгрузку продукции с отсрочкой платежей и снизить риски от внешнеэкономической деятельности, также сыграло немаловажную роль.

Отдельно стоит сказать о средних и малых предприятиях, подпадающих под критерии государственной поддержки. Такие бизнес-субъекты могут рассчитывать на расширение доступа к кредитным ресурсам. В разных странах к кредитованию таких бизнес-субъектов привлекаются и государственные банки, и коммерческие. В течение рассматриваемого периода (20072010 гг.) прямое кредитование бизнес-субъектов государственными банками практиковалось в следующих странах: Венгрия (объемы кредитования в 2010 году составили 1,1 млрд. долларов), Португалия, Южная Корея, Швеция (448,7 млн. долларов в 2009 году), Словакия (147 млн. евро в 2010 г.) и Италия.

Помимо прямого кредитования в рассматриваемых странах применялись и другие меры. Например, в Южной Корее и Италии широкое распространение получили программы государственных гарантий и пролонгация кредитов. В Португалии на время кризиса были введены кредитные линии сроком до семи лет. Также правительство разработало несколько пакетов по долгосрочному финансированию бизнес-субъектов. В общей сложности стоимость проекта составила около 9,7 млрд. евро. В некоторых странах были введены налоговые вычеты и предусмотрены налоговые льготы. Предприятия рассматриваемого сегмента получали финансирование за счет средств пенсионного фонда. При этом правительствами отдельных стран поиск дополнительных источников финансирования не прекращался в течение всего кризисного периода. Также менялись методы и подходы к определению того, что следует считать просроченной задолженностью. Ком- 
плексный подход позволил большинству субъектов среднего и малого бизнеса пережить кризисный период. Кроме того, были нивелированы неблагоприятные последствия, с которыми экономические агенты столкнулись в результате сокращения и ужесточения условий кредитования.

В контексте задач, поставленных правительствами стран-членов ЕАЭС и властями Российской Федерации, касающихся развития сегмента среднего и малого бизнеса, особым значением обладает анализ подходов к кредитованию механизмов, используемых иностранными государствами и международными организациями для обеспечения поддержки указанного сегмента в кризисных условиях. В странах ЕС удельный вес средних и малых предприятий достигает 70\%, в США и Японии - около 50-60\%, в Республике Беларусь - 30\%, в Азербайджане и Российской Федерации - 40-45\%.

После мирового финансового кризиса 2008 г. правительства всех стран ЕАЭС разработали пакет мер по поддержке бизнеса, в т.ч. малого. Эти меры периодически пересматриваются и корректируются в зависимости от потребностей. Несмотря на активную работу в направлении поддержки сегмента среднего и малого бизнеса, до сих пор существует немало факторов, сдерживающих развитие данного сегмента. К числу таких факторов можно отнести:

- отсутствие доступа к коммерческим кредитам;

- недостаточность инструментов и механизмов, сопровождающих коммерческое кредитование как один из источников финансирования хозяйственной деятельности средних и малых предприятий;

- не разработанность программы государственных гарантий и поддержки субъектов среднего и малого бизнеса при кредитовании;

- отсутствие или недостаточность льготной кредитной и финансовой поддержки экспортной деятельности.

В России, Беларуси и других странах-членах ЕАЭС льготное кредитование в период 2014-2016 гг. предоставлялось по отдельным направлениям, т.е. было дифференцированным. При этом по процентным ставкам имела место повышательная динамика [6].

Российская Федерация, Армения, Казахстан, Беларусь и Кыргызстан имеют экономику транзитивного типа. Объемы кредитования в этих странах относительно низкие. Во многом это связано с тем, что в период глобального финансового кризиса процентные ставки по кредитам были слишком высокими. Особенность стран с транзитивной экономикой - активное проявление в периоды финансового кризиса девальвационных и инфляционных процессов. В результате повышаются риски по кредитам и растут процентные ставки. Страны с развитой экономикой в разгар кризиса сталкиваются с замедлением роста цен и дефляцией. Закономерным результатом этого становится снижение процентных ставок по кредитам.

В иностранных государствах кредитование средних и малых предприятий в периоды финансового кризиса реализуется с участием не только государственных банков развития, но и коммерческих финансовых структур, участвующих в программах, разработанных на правительственном уровне. По поводу стран-членов ЕАЭС отметим, что в каждой из них существует своя национальная программа по поддержке кредитования рассматриваемого бизнес-сегмента [12].

В Беларуси к кредитованию рассматриваемого сегмента правительство привлекает лизинговые компании и партнерские банки. Это предусмотрено и в национальной программе поддержки кредитования. Механизм кредитования в стране реализуется на двух уровнях. Национальный Банк развития (первый уровень) обеспечивает финансовыми ресурсами банки партнеры (второй уровень), которые работают непосредственно с заемщиками (принимают заявления на кредит, рассматривают заявки, отсеивают заемщиков, принимают окончательное решение о выдаче кредитов).

Проанализировав иностранный опыт кредитования субъектов среднего и малого бизнеса, видим, что именно в периоды рецессии и кризисов правительства наиболее активно используют разного рода гарантийные схемы и увеличивают объемы кредитования. Также именно в кризисные периоды в практику внедряются новые механизмы и инструменты, применение которых позволяет значительно уменьшить стоимость гарантийных схем, обеспечить применение венчурного финансирования и государственных гарантий по ссудам, выдаваемым на срок менее 12 месяцев.

В нашей стране обеспечением государственных гарантий при кредитовании рассматриваемого сегмента и повышением доступности кредитных ресурсов занимается $\mathrm{AO}$ «Корпорация МСП». В настоящее время для решения указанных задач реализуется Программа стимулирования субъектов среднего и малого бизнеса [10]. Целью этой Программы выступает создание эффективного механизма поддержки субъектов среднего и малого бизнеса за счет предоставления им оборотных кредитов (на пополнение оборотного капитала); льготных кредитов для приобретения и создания основных средств. К слову, льготные кредиты доступны также экспортно-кредитным предприятиям, товарная продукция которых выпускается на территории РФ. По- 
мимо указанного института развития, в сегменте среднего и малого бизнеса с целью координации оказания средним и малым предприятиям государственной поддержки в регионах функционирует 80 гарантийных фондов. Обеспечение государственными гарантиями в разных регионах страны находилось в кризисный период на уровне 50-70\% [9].

Из всех стран ЕАЭС наиболее ограниченными государственные гарантии являлись в Республике Беларусь. Только работая по приоритетным направлениям, предприятие могло рассчитывать на участие в соответствующих программах поддержки. Они могут рассчитывать на получение кредитов по ставке $5 \%$.

Российская Федерация, Армения, Казахстан, Беларусь и Кыргызстан могут перенять положительный опыт развития государственного экспортно-кредитного гарантирования и страхования, сложившийся в таких странах, как Португалия, Швеция, Дания и Южная Корея. Тогда средние и малые предприятия стран ЕАЭС смогут не только участвовать в программах по льготно- му экспортному кредитованию, но и получат гарантии возврата денег от экспортно-кредитного агентства [7].

Исследование показало, что наиболее проблемными аспектами в сфере развития сегмента среднего и малого бизнеса выступают: отсутствие должной финансовой поддержки предприятий-экспортеров; неразвитость гарантийного кредитования; ограниченный доступ субъектов среднего и малого бизнеса к кредитным ресурсам [8].

В условиях рыночной экономики сегмент среднего и малого бизнеса играет важную роль в развитии национальной экономики. Сказанное в полной мере применимо и к России. Нужно активизировать работу в направлении создания и внедрения в практику мер упреждающего характера по улучшению существующего климата для развития субъектов среднего и малого бизнеса. Если соответствующие шаги так и не будут предприняты, то могут возникнуть негативные последствия (вплоть до оттока бизнес-субъектов за рубеж, где созданы более благоприятные условия).

\section{ЛИТЕРАТУРА}

1. Аджиева А.Ю., Губенко Д.С. Роль кредитования в развитии малого и среднего предпринимательства // Эпомен. 2020. № 48. С. 21-29.

2. Анненков К.П., Васильева О.Н., Венгеровский Е.Л., Кравчук Е.Д., Исмаилов И.Ш., Ручкина Г.Ф., Шамсуваров А.Т. Банковское кредитование. Учебное пособие / Под ред. Г.Ф. Ручкиной. Москва, 2020.

3. Бабич С.Г. Современные тенденции в сфере кредитования малого и среднего предпринимательства в Российской Федерации // В сборнике: Вестник кафедры статистики Российского экономического университета имени Г.В. Плеханова. Статистические исследования социально-экономического развития России и перспективы устойчивого роста. Москва, 2019. С. 15-19.

4. Басков Д.В. Анализ и оценка кредитного риска в процессе банковского кредитования малого и среднего предпринимательства в цифровой экономике // В книге: Влияние цифровой экономики на финансовые рынки и банки. Под ред. В.Е. Косарева. Москва, 2020. С. 96-101.

5. Дахненко С.С., Венгеровский Е.Л. Некоторые вопросы совершенствования действующего законодательства в сфере финансовой поддержки и кредитования субъектов малого и среднего предпринимательства // Теория и практика общественного развития. 2020. № 1 (143). С. 111-116.

6. Ерохина Е.А., Рыжова 0.А. Анализ и оценка состояния кредитования малого и среднего предпринимательства // В сборнике: Наука. Образование. Инновации. сборник научных трудов по материалам V Международной научно-практической конференции. 2019. С. $16-24$.

7. Жирнель Е.В. Особенности российского рынка кредитования малого и среднего предпринимательства на современном этапе // Управленческое консультирование. 2020. № 11 (143). С. 77-92.

8. Панкова А.С. Кредитование субъектов малого и среднего предпринимательства в Центральном федеральном округе // В сборнике: В мире научных открытий. Материалы III Международной студенческой научной конференции. 2019. С. 143-146.

9. Сазонов А.И. 0 перспективах развития института кредитования в России: социальный инвестиционный кредит как инструмент поддержки малого и среднего предпринимательства // В книге: Право и бизнес: правовое пространство для развития бизнеса в России. Коллективная монография. В 4-х томах. Отв. редактор С.Д. Могилевский [и др.]. Москва, 2020. С. 430-437.

10. Татарников А.А., Щекочихина С.В. Механизм беспроцентного кредитования субъектов малого и среднего предпринимательства как фактор инновационного и структурного развития экономики России // Самоуправление. 2020. № 5 (122). С. 452-455.

11. Шершнева Ю.А. Государственная политика поддержки малого и среднего предпринимательства // Вестник Коми республиканской академии государственной службы и управления. Теория и практика управления. 2019. № 22 (27). С. 81-83.

12. Шеина Е.Г., Серебрякова А.Ю. Проблемы кредитования сектора малого и среднего предпринимательства в Российской Федерации // Российское предпринимательств0. 2019. Т. 20. № 1. С. 39-58.

(c) Шайдуллина Венера Камилевна (VKShajdullina@fa.ru).

Журнал «Современная наука: актуальные проблемы теории и практики» 